\title{
Real-Time Automatic ECG Diagnosis Method Dedicated to Pervasive Cardiac Care
}

\author{
Haiying ZHOU ${ }^{1}$, Kun-Mean HOU ${ }^{2}$, Decheng ZUO ${ }^{1}$ \\ ${ }^{1}$ School of Computer Science \& Technology, Harbin Institute of Technology, Harbin, China \\ ${ }^{2}$ LIMOS Laboratory UMR 6158 CNRS, University of Blaise Pascal, Clermont-Ferrand, France \\ Email: \{haiyingzhou,zdc\}@hit.edu.cn, kun-mean.hou@isima.fr \\ Received May 1, 2009; revised May 25, 2009; accepted May 31, 2009
}

\begin{abstract}
Recent developments of the wireless sensor network will revolutionize the way of remote monitoring in different domains such as smart home and smart care, particularly remote cardiac care. Thus, it is challenging to propose an energy efficient technique for automatic ECG diagnosis (AED) to be embedded into the wireless sensor. Due to the high resource requirements, classical AED methods are unsuitable for pervasive cardiac care (PCC) applications. This paper proposes an embedded real-time AED algorithm dedicated to PCC systems. This AED algorithm consists of a QRS detector and a rhythm classifier. The QRS detector adopts the linear time-domain statistical and syntactic analysis method and the geometric feature extraction modeling technique. The rhythm classifier employs the self-learning expert system and the confidence interval method. Currently, this AED algorithm has been implemented and evaluated on the PCC system for 30 patients in the Gabriel Monpied hospital (CHRU of Clermont-Ferrand, France) and the MIT-BIH cardiac arrhythmias database. The overall results show that this energy efficient algorithm provides the same performance as the classical ones.
\end{abstract}

Keywords: Pervasive Cardiac Care, Automatic ECG Diagnosis, QRS detector, Rhythm Classifier, Wireless Sensor Networks

\section{Introduction}

Due to the increasing occurrence of sudden death events caused by cardiovascular diseases, there is a need to provide a long-term, real-time continuous PCC service for the sudden death high-risk population. The PCC system has thus been developed for different populations at a variety of environment, including at home, clinical and outdoor.

The studies of AED methods focused mainly on the clinical services. Unlike the clinical applications, the acquisitions of the PCC system is ambulatory ECG signal that is non-stationary and easy-disturbed by interferences. Moreover, the nodes of the PCC system have strict resource constraints, i.e. the capacities of computation, storage and power supply. Classical AED algorithms are thus unfit for the PCC system.

Supported by Doctoral Fund of Youth Scholar of Ministry of Education of China (No.200802131024), French Program of Cooperation with China (No.20974WG), and Scientific Research Fund of Returned Oversea Scholars of Harbin city of China (No.RC2009LX010001).
This paper presents a real-time and low resource consumption AED algorithm for the PCC system. Section 2 introduces the state-of-the-art of the AED algorithms. Section 3 describes this algorithm in detail and section 4 presents the performance evaluation. The conclusions are drawn at the last section.

\section{State-of-the-Art}

Due to its high potential amplitude, steep slope (R-wave) and wide duration, QRS complex is generally used for the cardiac event diagnosis and analysis. Different AED algorithms are classified by Köhler et al. [1]: 1). Timedomain analysis can implement a simple and rapid detection but it is noise-sensitive; 2). Wavelet transform analysis has high detection performance but has huge computation overhead; 3). Syntax analysis exposes the wave pattern elements and their mutual relations, but it is noise-sensitive and has huge computations; 4). Neural network analysis needs a large amount of training sample set and long training time. 
Other classical AED techniques include: template matching [2], hidden Markov model [3], Hilbert transform [4], mathematical morphology [5] method, etc. These techniques generally have huge computation overhead. The new AED algorithms generally integrate multiple techniques. For example, Oliveira et al. [6] integrates the Hilbert transform and wavelet transform, and Szilágyi et al. [7] combines the neural network, wavelet transform and genetic algorithm techniques. Generally, these hybrid methods can improve the detection accuracy, but have huge computation overhead, more resource consumption and less operation efficiency.

\section{AED Algorithm}

\subsection{Signal Preprocessing and Conditioning}

Due to the non-stationary and easy-disturbed natures of the ambulatory ECG signals, the acquisitions of PCC system must be de-noised before making detection. Most of artifacts, such as baseline shift, electrical noise and muscle tremor interference, can be effectively eliminated or reduced by choosing suitable filters. In this subsection, we present the filter techniques.

\subsubsection{ECG Time Series}

There are three ECG signals series, i.e. $\mathrm{R}(\mathrm{t}), \mathrm{AD}(\mathrm{t})$ and $\mathrm{RC}(\mathrm{t})$, in our algorithm. The $\mathrm{R}(\mathrm{t})$ series is the raw ECG signals acquired from electrodes. It's generally contaminated by different kinds of noises. The $\mathrm{AD}(\mathrm{t})$ series is the adaptive differential signals with the processing of the differential filter and the adaptive filter. The inferences of the baseline drift and the motion artifacts can be eliminated in the $\mathrm{AD}(\mathrm{t})$ series; hence this series is used to detect and to localize the QRS complexes. The RC(t) series is the de-noised ECG signals with the operations of the band-pass filter and the linear amplifier. Since the electrical noises and the muscle tremors have been removed from the $\mathrm{RC}(\mathrm{t})$ series, hence it is used to extract the characteristics of the QRS complexes.

\subsubsection{Adaptive Filter}

The classical filter for the ECG series, e.g. Notch filter, low-pass filter, and high-pass filter, can effectively remove or reduce most of the interferences. But for the motion artifacts, because of their irregular occurrences and irregular morphological attributes, these filters cannot eliminate these disturbances. These artifacts can make greatly troubles in QRS detection when encountering QRS-like artifacts.

This algorithm adopts an adaptive filter to reduce motion artifacts. The resultant signal series, named $\mathrm{A}(\mathrm{t})$, are generated by performing AT operation in the raw series $\mathrm{R}(\mathrm{t})$. The expression of adaptive filter is

$$
\left\{\begin{array}{l}
\operatorname{Aecg}(0)=R(0) \\
\quad 0<\alpha<1, t=1 \cdots N \\
\operatorname{Aecg}(t)=\alpha * \operatorname{Aecg}(t-1)+(1-\alpha) * R(t)
\end{array}\right.
$$

were $\alpha$ s the balance coefficient that is relative to the signal sample frequency (default 0.95).

Figure 1 shows different ECG series. Figure 1(a) is the raw signals $\mathrm{R}(\mathrm{t})$ which are serious polluted by noises. Figure 1(b) represents the reconstructed series $\mathrm{RC}(\mathrm{t})$ when filtering the $\mathrm{R}(\mathrm{t})$ series by the classical filters, i.e. Notch filter, low-pass filter and high-pass filter. The $\mathrm{RC}(\mathrm{t})$ series still contain the interferences generally caused by baseline wandering and motion artifacts. Figure 1(c) is the adaptive filter signal A(t) when filtering the $\mathrm{R}(\mathrm{t})$ series by the adaptive filter, which has better signal quality than $\mathrm{RC}(\mathrm{t})$. Figure $1(\mathrm{~d})$ is the reconstructed signal $\mathrm{RC}^{*}(\mathrm{t})$ based on the adaptive filter signal $\mathrm{A}(\mathrm{t})$. Obviously, in contrast to the previous reconstructed signal $\mathrm{RC}(\mathrm{t})$, the signal $\mathrm{RC}^{*}(\mathrm{t})$ has better signal quality in which the motion artifacts are effectively eliminated.

\subsection{QRS Complex Detection}

This paper presents a new QRS detector which copes with noises, artifacts and variability of ECG morphology by exploiting a self-adaptive threshold method (SAT), and a particular state transition recognition procedure (STR). The SAT method is used to estimate the peaks of ECG sub-segments and the means of contextual thresholds, which allows estimating the optimum thresholds in segment space. The STR procedure traces the waveform changes of signal series and identifies QRS complexes based on the optimum thresholds and the rules of state transition.

\subsubsection{Diagnostic Segment Window (DSW)}

A short-term redundant data (default 5 seconds) is important in QRS detection. Firstly, this short-term segment

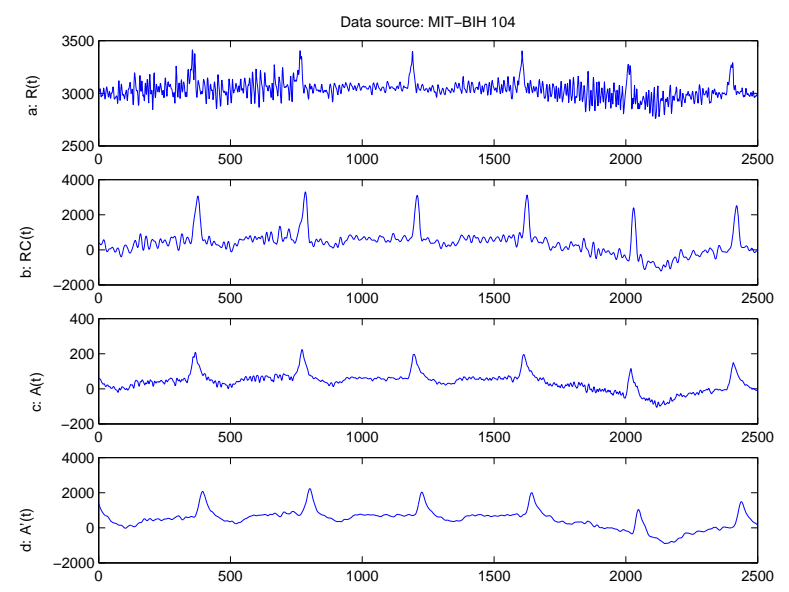

Figure 1. Filtered ECG signal series. 
enables the complex contextual correlative analysis and reduces the interferences of baseline drift. In view of the low-frequency baseline drift, a short-term segment has fewer disturbances caused by baseline wandering than long-term signals. The redundant data enable the QRS detector to identify current QRS complex by comparing with the fore-and-aft QRS complexes. Furthermore, in views of the unpredictability and variability of network quality, the redundancy is necessary for the data retransmission and the network communication.

\subsubsection{Self-Adaptive Threshold (SAT)}

The QRS waveforms in AECG have rapid changes and high potential amplitudes so that the differential series $D(\mathrm{t})$ can exactly represent the changes. The QRS signals have higher absolute amplitudes in a cardiac cycle of $D(\mathrm{t})$ series. The goal of QRS detection is to search the optimum pair-peak for each QRS complex, i.e. the positive and negative peaks of a cardiac cycle. In DSW, there are generally multiple pair-peaks because several heart beats will occur during the $5 \mathrm{~s}$ length. These pair-peaks make up of a pair-peak group in a DSW. Based on the pairthreshold obtained from the pair-peaks group of a DSW, the STR procedure is then able to locate QRS complexes. The absolute amplitude of each peak is generally greater than the associated absolute threshold in $D(\mathrm{t})$. Furthermore, since the offset of location between the $D(\mathrm{t})$ series and the $A(t)$ series is constant, we can thus obtain the positions of QRS complexes in $A(\mathrm{t})$ by locating the complexes in $D(\mathrm{t})$.

The SAT method aims to determine the optimum pairthreshold, which is estimated from two aspects: the mean of the pair-peak group of DSW and the pair-threshold of the previous DSW. The pair-threshold results from the means of the negative and positive pair-peaks group of DSW. In order to accurately estimate these pair-peaks, the diagnostic segment window is divided into 5 subsegments with the length of one second (see in Figure 2). Because the normal heart rate of a healthy adult is 60bmp-100bmp [8], each sub-segment thus contains one

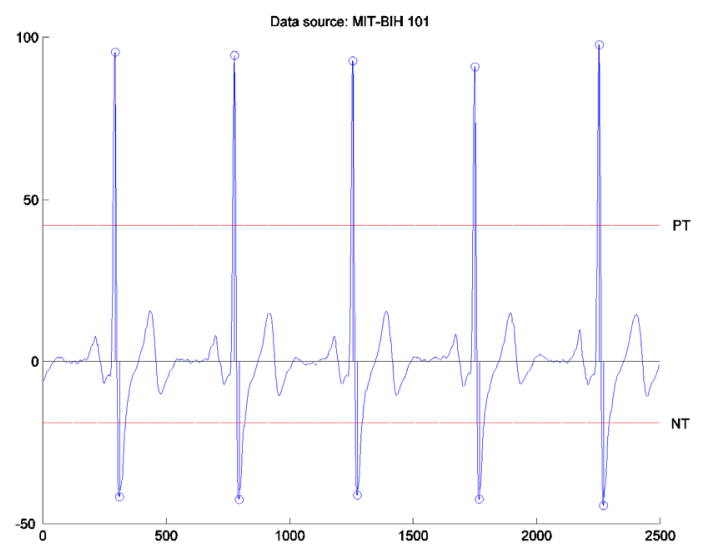

Figure 2. Mean of pair-peak group in diagnostic window. heart beat. Since the differential signals of QRS complex have the maximum absolute amplitudes in a cardiac cycle, a pair-peak will represent a QRS complex and then can be used to estimate the thresholds. Furthermore, the shorter of sub-segment is, the less interference of baseline drift the sub-segment has. A sub-segment with the length of one second can thus be regarded as a stationary series.

\subsubsection{QRS Location: State Transition Recognition}

In view of the QRS morphology properties in $D(\mathrm{t})$ series, the complexes are categorized into two groups: positive and negative. Therefore, the different states are defined to outline the phases of QRS complex in $D(\mathrm{t})$. S2 S9 represent the positive states of QRS complex (see in Figure 3), corresponding S20-S29 represent negative states. An adaptive and self-corrected procedure, named STR (State Transition Recognition), is developed to automatically track the changes of signal series, to correct error detection and to record detected complexes. The states transitions are based on three basic reference lines: the baseline, the positive threshold and the negative threshold.

\subsubsection{Feature Extraction: Geometric Analysis Method} QRS complex has the triangular-alike or triangularcomponent morphological characteristics, see in Figure 4. This paper thus employs the geometric analysis method (GAM) to extract the features of QRS complexes. GAM has simple operations and low resource consumption, being able to predict and estimate the key points of QRS complexes under noisy situations, such as $\mathrm{R}$ wave peak, end point of $\mathrm{Q}$ wave $(\mathrm{Qt})$ and onset point of $\mathrm{S}$ wave $(\mathrm{Si})$. Therein, $\mathrm{R}$ wave peak is obtained from Tpeak1 or Tpeak2 and it has mono-peak or poly-peaks. The measurement and the detection phases of Qt and Si points are illuminated as follows.

- Define two-level thresholds for left and right sides of $\mathrm{R}$ wave $(\mathrm{LH}=1 / 4 *$ Vpeak1, $\mathrm{LL}=3 / 4 *$ Vpeak1, $\mathrm{RH}=$ $1 / 4 *$ Vpeak2 and $\mathrm{RL}=3 / 4 *$ Vpeak2).

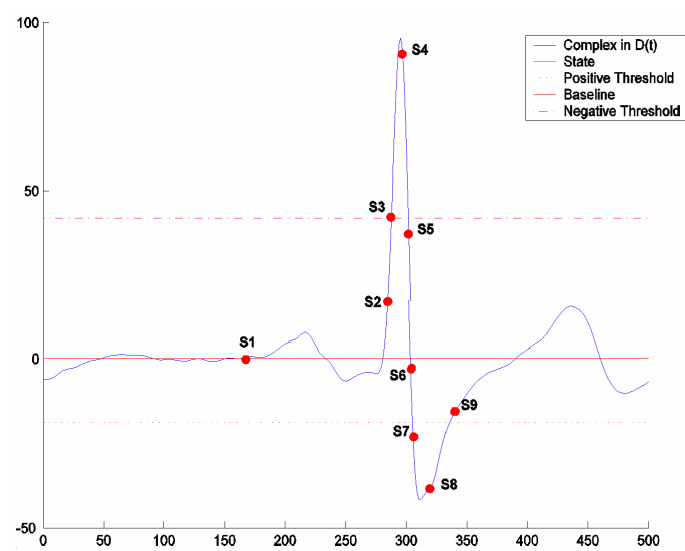

Figure 3. Positive states of QRS complex in $D(t)$. 


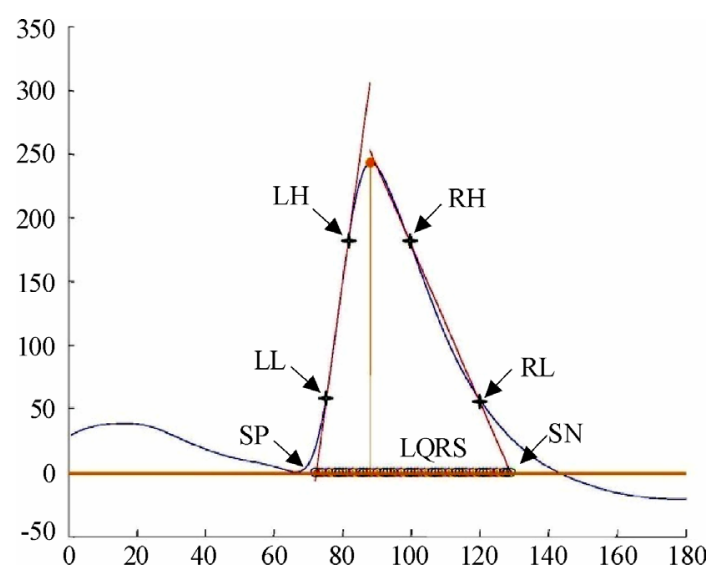

Figure 4. Illumination of geometric analysis method.

- Calculate the intersection points between the threshold values and complex signals. The slopes of two approaching lines represent two characteristics of QRS complex: SP (Positive Slope) and SN (Negative Slope).

- Obtain the duration length of QRS (LQRS) which is the distance of two intersection points between the baseline and two approaching lines.

\subsection{Cardiac Arrhythmias Classification}

Basing on the features values extracted from ECG signals, a self-diagnosis expert system is implemented to classify heart rhythms and interpret cardiac arrhythmias. The diagnostic rules of the expert system rely on the experiential rules estimated from the self-learning of system and the definitions of cardiologists. The diagnosis system is composed of three phases: a pre-learning machine, a rhythm classifier and an arrhythmia interpreter, see in Figure 5.

Based on the well-known experiential rules of cardiologists and the results of the training procedure, the prelearning machine builds and estimates the diagnostic rules for every lead ECG signals of a patient. The rhythm classifier classifies each detected heart rhythm into one of two catalogues: known rhythm or unknown rhythm. For the known rhythms, they are still classified into two types according to the values of the RR intervals: sinus rhythm and ventricular rhythm; and for the unknown rhythms, we will adopt classical methods to classify, the classification results will be verified by the cardiologists. In terms of the known rhythm types and the diagnostic rules, the cardiac arrhythmias interpreter is used to explain cardiac arrhythmias with the symptoms of relative heart diseases.

\subsubsection{Automatic Learning Machine}

Ten seconds ECG signals are used to calculate the rhythm template and to estimate the diagnostic rules. The

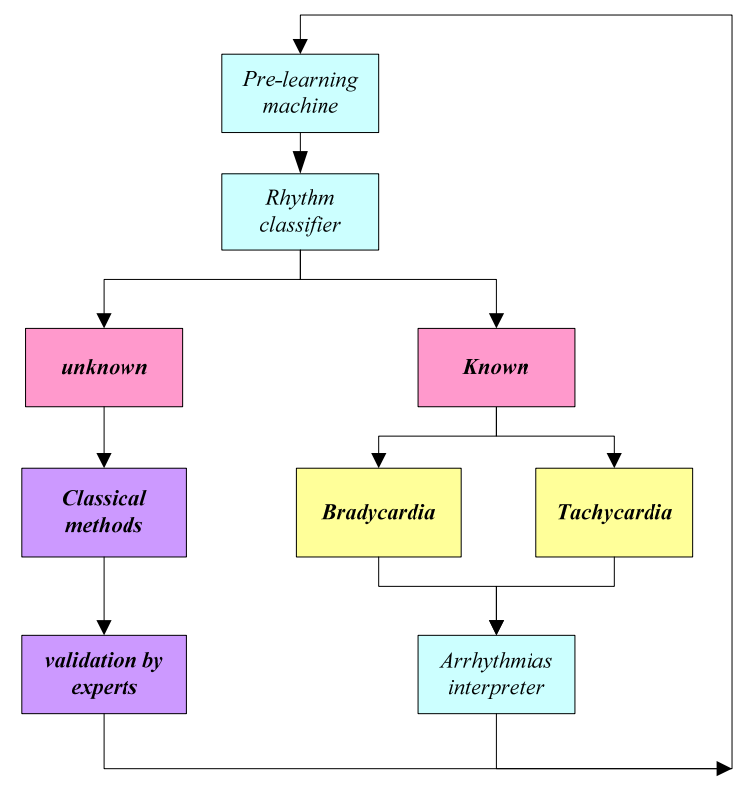

Figure 5. Illumination of automatic diagnosis system.

initial cardiac status, rhythm type, statistical and morphological features are achieved in this module. The diagnostic results will be further fed back to adjust the coefficients of diagnostic rules. Unlike resting ECG, long-term ambulatory ECG has continual tiny changes with the influences of exterior environments and the patient's physical status. The tiny changes are generally normal and the coefficients of diagnostic rules thus should be self-updatable to meet the changes.

\subsubsection{Rhythm Classifier}

By adopting the expert system and the confidence interval method, the rhythm classifier can recognize two kinds of QRS complex rhythms: sinus and ventricular. The details of signal features (RR interval, QRS duration, $\mathrm{R}$ wave left- \& right-sides slopes, $\mathrm{R}$ wave amplitude, and QRS absolute area), the rhythm type and the complex peaks are used to describe a heart rhythm. Hence, they can be used to recognize a rhythm and by comparing with the features of the rhythm template.

The rhythm classifier is based on the features comparison and the interval estimation. Since we have obtained the features of current rhythm and the features of the standard rhythm (rhythm template) in pre-learning machine, the rhythm classification is thus to estimate the confidence intervals, the weighed factors and the deviation coefficients of the features. The classification equation can be expressed as:

$$
\rho=\sum_{i=1}^{N} \rho_{i}=\sum_{i=1}^{N}\left(\beta_{i} * \alpha_{i}\right)
$$

Where the $\rho_{i}$ is the classification factor that is used to determine the heart rhythm by estimating the confidence 
interval that it falls; the $\beta_{i}$ is the weighed factor that indicates the contribution of the feature $i$; the $\alpha_{i}$ is coefficient of deviation that associates the variation of the feature $i$; the $N$ indicates the number of the features.

\subsubsection{Arrhythmia Interpreter}

In terms of the rhythm type and heat rate (HR), a heart rhythm can be recognized and interpreted by the arrhythmia interpreter basing on diagnostic rules. Firstly, the arrhythmia interpreter classifies the rhythms into two catalogues: bradycardia and tachycardia by comparing current HR with the mean HR of the rhythm template.

In arrhythmia interpreter, the heart rhythms are identified and classified into two basic categories: normal cardiac rhythms and cardiac arrhythmias, and the cardiac arrhythmias can be further divided as two classes: the known cardiac arrhythmias and the unknown cardiac arrhythmias. The known cardiac arrhythmias are normally the cardiac tachycardia events which are caused by serious heart diseases, including PVC (Premature ventricular complexes), VT (Ventricular tachycardia), VF (Ventricular Fibrillation), SVT (Supraventricular Tachycardia) and PAC (Premature Atrial Contraction), etc. The known cardiac arrhythmias have distinctive QRS complexes and rapid heart rates which make them be interpreted accurately. The unknown cardiac arrhythmias are normally the bradycardia events which are caused by less serious or benign heart diseases. The known cardiac arrhythmias have regular heart rhythms but slow heart rates, the identifications of which are not reliable when depending only on the heart rate.

\section{Performance Analysis}

The algorithm has been assessed on two ECG databases: MIT-BIH arrhythmia database [9] and CSD database (Clinic STAR Database). The former contains 48 halfhour excerpts of two-channel ambulatory ECG recordings, and the latter is obtained from 30 subjects of the Gabriel Montpied hospital (CHRU de ClermontFerrand, France) by using a PCC system named STAR [10]. The CSD signals are recorded in the same format (WFDB) as MIT-BIH Database one.

\subsection{STAR System}

Currently, a real-time remote continuous cardiac arrhythmia detecting and monitoring system, named STAR (Système Télé-Assistance Réparti), has been developed by the SMIR group of LIMOS laboratory of the Blaise Pascal University and been applied on the CHU de Gabriel Montpied hospital (Clermont-Ferrand, France). The STAR system combines the technology advantages of pervasive computing, AED algorithm and remote telemedicine system. Figure 6 shows its system architecture, which consists of local wireless ECG sensor (WES) nodes and remote cardiac surveillance system.

The system description is: a WES device equipped by the surveillance object, which integrates the AED algorithm, can acquire and analyze the patient's ECG signals in real-time. When a cardiac abnormal event is detected, an alarm message and (or) a segment of ECG signals will send to the cardiologists via the available wired or wireless communication mediums. In the remote cardiac surveillance system, the cardiologists can examine cardiac abnormal events by employing AED algorithm and make a respond with the shortest delays. This system aims to provide a rapid detection and diagnosis method for the high-risk population of cardiac arrhythmias to prevent sudden death. It is also used to do long-term heart surveillance for the population who has the history of heart diseases, or to do periodic heart examination for the health population.

\subsection{QRS Detector Evaluation}

Dotsinsky et al. [11] defined four performance parameters to assess the algorithm efficiency (Se: sensitivity and Sp: specificity): TP (true positive), FP (false positive), FN (false negative) and shifted SH beats, shown as follows:

$$
\begin{gathered}
S e=\frac{T P}{T P+F N+S H} \\
S p=1-\frac{F P}{T P+F P}=\frac{T P}{T P+F P}
\end{gathered}
$$

Comparing with the performance results of other algorithms listed in Table 1, the performance results of this

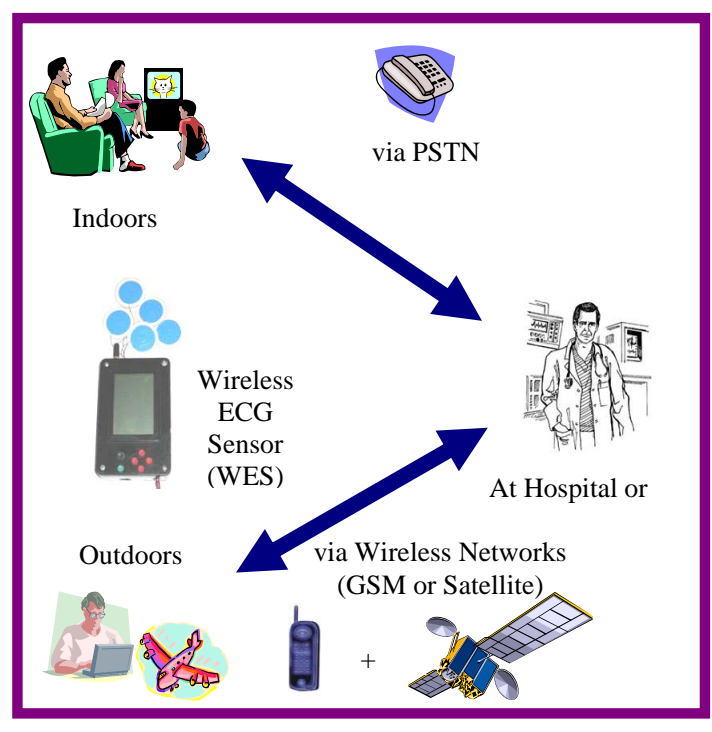

Figure 6. Architecture of STAR system. 
Table 1. Performance evaluation of QRS detection algorithms.

\begin{tabular}{|c|c|c|c|c|c|}
\hline & & \multicolumn{2}{|c|}{$\mathrm{Se}(\%)$} & \multicolumn{2}{|c|}{ Sp(\%) } \\
\hline \multicolumn{2}{|l|}{ Afonso et al [12] } & \multicolumn{2}{|c|}{99.59} & \multicolumn{2}{|c|}{99.56} \\
\hline \multicolumn{2}{|l|}{ Poli et al [13] } & \multicolumn{2}{|c|}{99.60} & \multicolumn{2}{|c|}{99.51} \\
\hline \multicolumn{2}{|c|}{ Dotsinsky et al [11] } & \multicolumn{2}{|c|}{99.04} & \multicolumn{2}{|c|}{99.62} \\
\hline \multicolumn{2}{|l|}{ Kaiser et al [14] } & \multicolumn{2}{|c|}{99.68} & \multicolumn{2}{|c|}{99.72} \\
\hline \multicolumn{2}{|c|}{ Datex-Ohmeda Corp. [16] } & \multicolumn{2}{|c|}{99.86} & \multicolumn{2}{|c|}{99.88} \\
\hline \multirow{2}{*}{ Millet et al [15] } & Alg 1 & \multicolumn{2}{|c|}{94.6} & \multicolumn{2}{|c|}{98.0} \\
\hline & Alg 2 & \multicolumn{2}{|c|}{97.3} & \multicolumn{2}{|c|}{98.0} \\
\hline \multicolumn{2}{|l|}{ Our Algorithm } & $\begin{array}{c}99.43 \\
\text { MIT }\end{array}$ & $\begin{array}{c}99.25 \\
\text { CSD }\end{array}$ & $\begin{array}{c}98.55 \\
\text { MIT }\end{array}$ & $\begin{array}{l}97.94 \\
\text { CSD }\end{array}$ \\
\hline
\end{tabular}

detection algorithm, 99.37\% sensitivity and 99.68\% specificity on MIT-BIH database, $99.67 \%$ sensitivity and 99.74\% specificity on CSD database, show the high sensitivity and specificity. This detection algorithm has minimal beat detection latency, low computational consumption and fast detection ability.

\subsection{Rhythm Classifier Evaluation}

The rhythm classifier classifies heart rhythms into two catalogues: non-alarm and alarm-rhythms. The alarmrhythms defined in our algorithm are tachycardia, i.e. PAC, PVC, SVT, VT, and VF. They represent serious heart diseases which need to be reported immediately. The non-alarm rhythms include the normal rhythms and some benign or less serious cardiac arrhythmias, such as bradycardia.

The four parameters are used to assess the algorithm performance [17]: A true positive (TP) is a serious cardiac arrhythmia that has been correctly classified as an alarm- rhythm; A false positive (FP) is an organized normal rhythm that has been incorrectly classified as an alarm- rhythm; A true negative (TN) is any normal or less serious rhythm that has been correctly classified as a non-alarm rhythm; A false negative (FN) is a serious cardiac arrhythmia that has been incorrectly classified as a non-alarm rhythm.

The sensitivity (Se) is the number of true positive abnormal rhythms, expressed as a percentage of the total number of abnormal rhythms. Se is calculated by formula (3). The specificity (Sp, also named positive predictive accuracy) is the number of organized rhythms that have been correctly classified as normal rhythms, expressed as a percentage of the total number of normal rhythms and computed by formula (4).

$$
S p=\frac{T N}{F P+T N}
$$

Comparing with the performance of other algorithms listed in Table 2, the performance results of this classification algorithm, $90.90 \%$ sensitivity and $95.50 \%$ specificity on MIT-BIH database, $95.6 \%$ sensitivity and 99.5\% specificity on CSD records, show its good performance. Since the features extracted by the detection algorithm are the time domain characteristics of QRS complex, this classification algorithm thus can directly utilize the experiences of cardiologists that reduces the complexity of rules training and then improves the accuracy of classification. Another advantage is that this algorithm is able to identify various cardiac arrhythmias comparing to most of other algorithms.

\section{Conclusion}

The objective of our research is to design a real-time energy efficient Automatic ECG Diagnosis algorithm for the PCC system. The PCC application is free of the limitations of time and space, that is, this system supports long-term monitoring (from few days to one month) and the patient have the freedom of daily actions. The results of the performance evaluation show that our algorithm satisfies application demands.

Table 2. Performance evaluations of rhythm classification algorithm.

\begin{tabular}{|c|c|c|c|c|c|c|c|c|c|c|c|c|c|c|}
\hline & \multicolumn{2}{|c|}{ NSR } & \multicolumn{2}{|c|}{ PAC } & \multicolumn{2}{|c|}{ PVC } & \multicolumn{2}{|c|}{ SVT } & \multicolumn{2}{|c|}{ VT } & \multicolumn{2}{|c|}{$\mathrm{VF}$} & \multicolumn{2}{|c|}{ Total } \\
\hline & Se & Sp & Se & Sp & Se & Sp & Se & Sp & Se & Sp & Se & Sp & Se & Sp \\
\hline Horácek [18] & & & & & & & & & 90.3 & 78.6 & & & & \\
\hline Ge et al [19] & 93.2 & 94.4 & 96.4 & 96.7 & 94.8 & 96.8 & 100 & 96.2 & 97.7 & 98.6 & 98.6 & 97.7 & 96.83 & 96.73 \\
\hline Ham \& Han [20] & & & & & 99 & 97 & & & & & & & & \\
\hline Chen et al [21] & & & & & & & & & 93 & & 96 & & & \\
\hline Minami et al [22] & & & & & $>98$ & $>98$ & & & $>98$ & $>98$ & & & & \\
\hline Melo et al [24] & & & 93 & & 99 & & & & & & & & & \\
\hline Datex-Ohmeda [16] & & & & & 94.08 & 97.55 & & & & & & & & \\
\hline Philips AED [17] & & & & & & & & & 84 & 91 & $97^{*}, 76^{* *}$ & 91 & & \\
\hline Our Algorithm & & & & & & & & & & & & & $\begin{array}{l}90.9^{1} \\
95.6^{2}\end{array}$ & $\begin{array}{l}95.5^{1} \\
99.5^{2}\end{array}$ \\
\hline
\end{tabular}

*:Ventricular Fibrillation (amplitude $>0.200 \mathrm{mv}) . \quad * *$ : Fine Ventricular Fibrillation $(0.100 \mathrm{mv}<$ amplitude $<0.200 \mathrm{mv})$

${ }^{1}$ Evaluation results on MIT-BIH database $\quad{ }^{2}$ Evaluation results on CSD clinical records 
In this AED algorithm, the QRS detector adopts linear time-domain statistical analysis and syntactic analysis methods to locate QRS complex from AECG signals. The signal preprocessing and conditioning procedure, adopting adaptive filter and band-pass filter, remove or reduce various interferences caused by physical and technical factors. The most serious noisy, such as motion artifacts, has been effectively eliminated by the adaptive filter. According to the statistical feature and morphologic features of QRS complex, i.e. heart rate, steep edges and sharp amplitude, the QRS complex is located to mark heart beat by applying SAT method and STR procedure on sub-segment diagnosis window.

The rhythm classifier classifies rhythms and interprets cardiac arrhythmias basing upon the diagnostic rules which are obtained from the experiences of cardiologists and the training results of pre-learning phase. The initial ECG signals with the length of 10 seconds are used to estimate the type of QRS complex and to extract the features of normal rhythm template (the means of LQRS, $\mathrm{RR}$, etc.). According to the origination of heart beat, the rhythms are categorized into two classes: sinus rhythm (atria) and ventricular rhythm (ventricle). According to the changes of heart rate, cardiac arrhythmias are categorized into two classes: bradycardia and tachycardia. The cardiac arrhythmias interpretation procedure is adopted to classify cardiac arrhythmias into various types of bradycardia and tachycardia, based on the features extracted in the detection algorithm.

Currently, this algorithm has been applied on the STAR system. The performance evaluations results show that this algorithm was effective for the QRS detection and the rhythm classification, and was thus suitable for PCC services. The simple, fast and efficient features of this algorithm enable it to be embedded into microprocessor system or be implemented on chip.

\section{Acknowledgement}

This project is supported by OSEO (French research agency) and the Conseil Régional d'Auvergne (France).

\section{References}

[1] B.-U. Köhler, C. Hennig, and R. Orglmeister, "The principles of software QRS detection,” IEEE Engineering in Medicine and Biology Magazine, Vol. 21, No. 1, pp. 42-57, 2002.

[2] J. M. Jenkins and S. A. Caswell, "Detection algorithm in implantable cardioverter Defibrillators," Proceedings of the IEEE, Vol. 84, No. 3, pp. 428-445, 1996.

[3] S. A. Coast, R. M. S tem et al., "An approach to cardiac arrhythmia analysis using hidden markov models,” IEEE Transaction On Biomedical Engineering, Vol. 37, No. 9, pp. 826-835, 1990.
[4] D. S. Benitez, P. A. Gaydecki, A. Zaidi et al., "A new QRS detection algorithm based on the Hilbert transform," IEEE Computers in Cardiology, pp. 379-382, 2000.

[5] P. E. Trahanias, “An approach to QRS complex detection using mathematical morphology,” IEEE Transaction On Biomedical Engineering, Vol. 40, No.2, pp. 201-205. 1993.

[6] F. I. de Oliveira and P. U. Cortez, "A QRS detection based on Hilbert transform and wavelet," Proceedings of 14th IEEE SPSW on MLSP, pp. 481-489, 2004.

[7] S. M. Szilágyi, Z. Benyo, L. Szilagyi, et al., "Adaptive wavelet transform based ECG waveforms detection," Proceedings of 25th Annual IEEE EMBS International Conference, No. 24, pp. 12-15, 2003.

[8] W. A. H. Engelse and C. Zeelenberg, "A single scan algorithm for QRS-detection and feature extraction, Computers in Cardiology,” No. 6, pp. 37-42, 1979.

[9] G. B. Moody and R. G. Mark, "The MIT-BIH arrhythmia database on CD-ROM and software for use with it," Computers in Cardiology, Vol. 17, pp. 185-188, 1990.

[10] H. Y. Zhou, K. M. Hou et al., "Remote continuous cardiac arrhythmias detection and monitoring," 2nd International Conference on E-health in Common Europe, Krakow, pp. 11-12. March 2004.

[11] I. A Dotsinsky and T. V Stoyanov, "Ventricular beat detection in single channel electrocardiograms," Biomedical Engineering Online, Vol. 3, No. 3, 2004.

[12] V. X. Afonso, W. J. Tompkins, T. Q. Nguyen and S. Luo, "ECG beat detection using filter banks," IEEE Transaction on Biomedical Engineering, Vol. 46: pp. 192-202. 1999.

[13] R. Poli, S. Cagnoni, and G. Valli, "Genetic design of optimum linear and nonlinear QRS detectors," IEEE Transaction On Biomedical Engineering, Vol. 42, pp. 1137-1141, 1995.

[14] W. Kaiser and M. Findeis, "Novel signal processing Methods for exercise ECG," Special issue on Electrocardiography in Ischemic Heart Disease, Proceedings of IJBEM, Vol. 2, 2000.

[15] J. Millet, M. Perez, G. Joseph, A. Mocholi, and J. Chorro, "Previous identification of QRS onset and offset is not essential for classifying QRS complex in a single lead," Computers in Cardiology, Vol. 24, pp. 299-302, 1997.

[16] Datex-Ohmeda Corp.: Bedside Arrhythmia Monitoring Quick Guide, Education Report, Internal web journal for medical professionals (Focus on Cardiovascular), URL: http://www.clinicalwindow.com, November 2002.

[17] Philips Medical systems: AED Algorithm application Note Philips HeartStart, Technical Document, URL: http://www.medical.philips.com, Philips Electronics North America Corporation, 2003.

[18] M. B Horácek, "Body-surface potential maps can identify substrate for ventricular arrhythmias," International Journal of Bioelectromagnetism," Vol. 5, No. 1, pp. 331-334, 2003.

[19] D. F. Ge, N. Srinivasan, and S. M. Krishnan, "Cardiac 
arrhythmia classification using autoregressive modeling," Biomed Eng Online, Vol. 1, No. 5, 2002.

[20] F. M. Ham and S. Han, "Classification of cardiac arrhythmias using fuzzy ARTMAP,” IEEE Trans Biomed Eng; Vol. 43, No. 4, pp. 425-30, April 1996.

[21] S. W. Chen, P. M. Clarkson, and Q. Fan, "A robust sequential detection algorithm for cardiac arrhythmia classification,” IEEE Trans Biomed Eng, Vol. 43, No. 11, 1120-5, November 1996.

[22] K. C. Minami, H. Nakajima, and T. Toyoshima, "Real-time discrimination of ventricular tachyarrhythmia with fourier-transform neural network,” IEEE Trans Biomed Eng, Vol. 46, pp. 179-185, 1999.

[23] S. W. Chen, "Two-stage discrimination of cardiac arrhythmias using a total least squares-based prony modeling algorithm,” IEEE Trans Biomed Eng, Vol. 47, pp. 1317-1326, 2000.

[24] S. L. Melo, L. P. Caloba, and J. Nadal, “Arrhythmia analysis using artificial neural network and decimated electrocardiographic data," Comp Cardiol, Vol. 27, pp. 73-76, 2000. 